\title{
The Magnocellular Mediodorsal Thalamus is Necessary for Memory Acquisition, But Not Retrieval
}

\author{
Anna S. Mitchell and David Gaffan \\ Department of Experimental Psychology, Oxford University, Oxford OX1 3UD, United Kingdom
}

\begin{abstract}
Damage to the magnocellular mediodorsal thalamic nucleus (MDmc) in the human brain is associated with both retrograde and anterograde amnesia. In the present study we made selective neurotoxic MDmc lesions in rhesus monkeys and compared the effects of these lesions on memory acquisition and retrieval. Monkeys learned 300 unique scene discriminations preoperatively and retention was assessed in a one-trial preoperative retrieval test. Bilateral neurotoxic lesions of the $\mathrm{MDmc}$, produced by $10 \times 1 \mu \mathrm{l}$ injections of a mixture of ibotenate and NMDA did not affect performance in the postoperative one-trial retrieval test. In contrast, new postoperative learning of a further 100 novel scene discriminations was substantially impaired. Thus, MDmc is required for new learning of scene discriminations but not for their retention and retrieval. This finding is the first evidence that MDmc plays a specific role in memory acquisition.
\end{abstract}

Key words: mediodorsal thalamus; prefrontal cortex; retrograde amnesia; episodic memory; new learning; primate

\section{Introduction}

Damage to the medial diencephalon in the human brain is associated with both retrograde and anterograde amnesia. On the basis of human neuropathology it is difficult to determine what sites of damage are critical in the production of diencephalic amnesia, but some evidence indicates that the processes underlying retrograde and anterograde amnesia may be independent of each other. Kopelman et al. $(1999,2003)$ have proposed that severe and temporally extensive retrograde amnesia is seen only when the diencephalic damage is accompanied by extensive cortical pathology, typically in clinical cases of Korskoff's syndrome. In contrast, patients with focal diencephalic lesions were reported to have little or no retrograde amnesia (Graff-Radford et al., 1990; Parkin et al., 1994; Kopelman et al., 1999) (but see Miller et al., 2001, 2003). These observations suggest that medial diencephalic structures may be involved selectively in acquiring new memories, rather than both memory acquisition and retrieval processes. One structure often damaged in clinical cases is the mediodorsal thalamus (Victor et al., 1989; Parkin et al., 1994; Harding et al., 2000; Miller et al., 2001, 2003) (but see Mair et al., 1979; Mayes et al., 1988). Studies in monkeys have shown that selective lesions to the magnocellular mediodorsal thalamic nucleus $(\mathrm{MDmc})$ produce anterograde amnesia in the form of postoperative learning impairments in many visual memory tasks (Aggleton and Mishkin, 1983; Zola-Morgan and Squire, 1985; Gaffan and Watkins, 1991; Gaffan et al., 1993; Parker et al., 1997; Parker and Gaffan, 1998; Gaffan and Parker, 2000; Mitchell et al.,

Received Sept. 27, 2007; revised Nov. 28, 2007; accepted Nov. 29, 2007.

This work was supported by the Medical Research Council, United Kingdom. We thank Dr. M. Baxter and Professor J. Aggleton for comments on a previous version of this manuscript, and M. Brown, P. Browning, G. Daubney, K. Murphy, and S. Mygdal for technical support.

Correspondence should be addressed to Dr. Anna S. Mitchell, Department of Experimental Psychology, Oxford University, South Parks Road, 0xford OX1 3UD, UK. E-mail: anna.mitchell@psy.ox.ac.uk.

DOI:10.1523/JNEUROSCI.4922-07.2008

Copyright $\odot 2008$ Society for Neuroscience $\quad$ 0270-6474/08/280258-06\$15.00/0 2007a), but their effects on retention of information acquired before neurosurgery have not been fully tested (Mitchell et al., 2007a). Thus, MDmc damage may not produce both the anterograde and retrograde amnesia characteristic of diencephalic amnesia.

Experimental verification of this hypothesis would not only clarify the neuropathological basis of diencephalic amnesia, but would also confirm whether the acquisition and retrieval of memories is performed by the same neural systems. Thus, we tested whether selective neurotoxic lesions of the MDmc in monkeys produce both retrograde and anterograde amnesia. Here, we report the first pure measurement of retrograde amnesia, uncontaminated by anterograde amnesia, after MDmc lesions in monkeys. Our test used the object-in-place scene discrimination problems that model the "what" and "where" aspects of episodic memory in monkeys (Gaffan, 1994).

We taught 300 unique scene discriminations to each monkey preoperatively, and then tested memory for those scenes in a preoperative and a postoperative retrieval test with only one trial for each of the 300 scenes. Because this one-trial test cannot be contaminated by any effect of postoperative reacquisition, it is a pure measure of memory retention and retrieval. After this test of retrograde amnesia, we assessed anterograde amnesia with a test of new postoperative learning of scene discriminations, requiring new learning of a set of 100 scenes over several days.

\section{Materials and Methods}

Subjects. Eight naive rhesus monkeys (Macaca mulatta), all male, 5.52$8.34 \mathrm{~kg}$ (between 2 and 3 years old) at the beginning of behavioral training, participated in this study. Three monkeys (MD4, MD5, and MD6) formed the MDmc lesion group. Five monkeys (CON1, CON2, CON3, CON4, and CON5) were unoperated controls. All three monkeys in the MDmc group, and four of the monkeys in the control group, performed the preoperative acquisition, the preoperative and postoperative onetrial retrieval tests, and a test of postoperative acquisition. The remaining control monkey, CON4, performed the preoperative acquisition and the 
preoperative and postoperative one-trial retrieval tests, but for reasons unconnected with the present study was not available for the test of postoperative acquisition. The same 300 unique scene discriminations were used for all of the monkeys in preoperative training. All experimental procedures were performed in compliance with the United Kingdom Animals (Scientific Procedures) Act of 1986.

Apparatus. The computer-controlled test apparatus was identical to that which has been described previously in detail by Mitchell et al. (2007a). Briefly, it consisted of a large touch-sensitive color monitor that displayed the visual stimuli and food reward dispensers.

Scene discrimination learning. The scene discrimination learning task was adapted from Gaffan (1994). The stimulus material was identical to those described in detail along with example stimuli (Mitchell et al., 2007a). Briefly, each trial consisted of an artificially constructed "scene." There were two foreground objects in each scene, one correct (rewarded) and the other incorrect (nonrewarded), consisting of randomly selected small colored typographic characters each placed in a constant location. Each scene was unique in that they varied in several randomly selected attributes including (1) the background color of the screen, (2) the location of ellipses on the screen, (3) the color, size, and orientation of ellipse segments, (4) the typographic character, clearly distinct in size from the foreground objects, and (5) the color of the typographic character. All of the colors were assigned with the constraint that the foreground objects should be visible (that is, there was a minimum separation in color space between the colors of a foreground object and the color of any element of its local background).

Behavioral training. A detailed explanation of pretraining is provided by Mitchell et al. (2007a). When the monkeys were reliably touching the foreground objects when presented with a new scene and completing 50 trials a day with minimal accuracy errors (i.e., touching any location on the screen other than the foreground typographic characters) behavioral training began.

The 300 unique scene discriminations were divided into three sets (A, $\mathrm{B}, \mathrm{C})$ of 100 discriminations each with only one set presented per training day. The presentation order was consistent throughout each cycle of six training days: A, B, A, C, B, and A. In this way, set A was presented three times, set $\mathrm{B}$ twice, and set $\mathrm{C}$ once within every cycle. For the first and second cycles monkeys saw only the first 50 and then only the second 50 unique scene discriminations from each of the three sets, respectively. After these $12 \mathrm{~d}$ of training, further cycles began using all 100 unique scenes from each of the three sets until learning reached a criterion set at $85 \%$ or above across all three presentations of set A within one cycle of six training days. A touch to the correct foreground object caused the object to flash for $2 \mathrm{~s}$ then a reward pellet was delivered into the hopper and the screen went blank. A touch to the incorrect object caused the screen to blank immediately, no reward was given and an intertrial interval imposed for $10 \mathrm{~s}$. A correction trial was then administered in which the scene was re-presented with only the correct object present. Touches anywhere else in the scene caused the screen to blank and the trial was repeated. When the monkey completed the final trial of a training day the lunchbox opened automatically, the monkey received the large food reward and was allowed time to eat before being returned to the home enclosure.

Experiment 1: one-trial preoperative and postoperative retrieval tests. After reaching the training criterion, monkeys were given a rest for $12 \mathrm{~d}$. Then the preoperative retrieval test was conducted. The first day consisted of "familiarization"; monkeys saw 100 trials using a simpler version of the task, one random typographic character was presented against a black background and the monkeys had to touch the character to receive a reward. Responses to anywhere else on the screen immediately ended the trial and it was represented after a $10 \mathrm{~s}$ delay. This familiarization ensured that the monkeys had not altered their motivation as a consequence of the extended break from testing. On the second day, the monkeys were presented with set A using the same testing methods experienced during preoperative training then on the third day set B was presented and on the fourth day set C. These four consecutive days of testing constituted the one-trial preoperative retrieval test. Monkeys were then matched based on their performance and assigned to the MDmc lesion or unoperated control group. MDmc monkeys were scheduled for surgery and the others for an equivalent period of rest. After "postoperative" recovery of at least $12 \mathrm{~d}$ all monkeys were tested on the one-trial postoperative retrieval test, which was identical to the preoperative retrieval test.

Experiment 2: acquisition of 100 novel scene discriminations. Before acquisition training began on 100 novel scenes all monkeys completed $20 \mathrm{~d}$ of within-session new scene learning (data not presented here) using the standard scene learning task described by Gaffan (1994). The novel 100 unique scene discriminations (set D) were presented to the monkeys in the same way as during preoperative acquisition, however in this experiment there was only one set with at least a $24 \mathrm{~h}$ delay between trials. The type of stimuli and rewards used in this experiment were identical to the retrieval experiment. From the first day of training monkeys saw all 100 novel scenes. Correction trials as described above were used when an error occurred. The dependent measure was the number of errors made to reach a learning criterion of $>85 \%$ correct across three consecutive trials.

Surgery. A more detailed description of the surgical methods and preoperative and postoperative drug treatments is provided in Mitchell et al. (2007b). Briefly, each monkey was sedated on the morning of surgery with both ketamine and xylazine then during surgery was intubated, mechanically ventilated and maintained deeply anesthetized using sevoflurane.

The monkey was placed in a stereotaxic head holder. After opening the skin, underlying galea and creating a right-sided D-shaped bone flap, the dura over the posterior part of the right hemisphere was cut and retracted to the midline. The splenium of the corpus callosum was cut in the midline with a glass aspirator. The tela choroidea was cauterized in the midline posterior and dorsal to the thalamus using a metal aspirator that was insulated to the tip. A stereotaxic manipulator holding a $10 \mu \mathrm{l} \mathrm{Ham}-$ ilton syringe with a blunt tipped 26-gauge needle was positioned above the posterior commissure at the midline using the third ventricle as a guide. Neurotoxic bilateral lesions to the MDmc were produced by $10 \times$ $1 \mu \mathrm{l}$ injections of a mixture of ibotenic acid $(10 \mathrm{mg} / \mathrm{ml}$; Biosearch Technologies, Novato, CA) and NMDA (10 mg/ml; Tocris, Bristol, UK) dissolved in sterile $0.1 \mathrm{M}$ PBS. The monkey brain atlas of Ilinsky and KultasIlinsky (1987) was used to calculate the intended lesion site coordinates. The needle was positioned for the first set of coordinates: anteroposterior (AP), $+5.2 \mathrm{~mm}$ anterior to the posterior commissure; mediolateral (ML), $\pm 1.2 \mathrm{~mm}$ lateral to the third ventricle; dorsoventral (DV), -4.0 $\mathrm{mm}$ (to compensate for the hole positioned $1 \mathrm{~mm}$ above the tip of the needle) ventral to the surface of the thalamus directly above the intended lesion site. Each injection was made slowly over $4 \mathrm{~min}$ and the needle was left in place for $\sim 4$ min before being moved to the next site. The needle was then repositioned for the second set of coordinates: AP, $+4.2 \mathrm{~mm}$; $\mathrm{ML}, \pm 1.5 \mathrm{~mm}$; DV,$-5.0 \mathrm{~mm}$. The third, fourth, and fifth sets of coordinates were as follows: $\mathrm{AP},+4.2 \mathrm{~mm}, \mathrm{ML}, \pm 1.5 \mathrm{~mm}$, and $\mathrm{DV},-3.0$ $\mathrm{mm}$; AP, $+3.4 \mathrm{~mm}, \mathrm{ML}, \pm 1.7 \mathrm{~mm}$, and $\mathrm{DV},-4.0 \mathrm{~mm}$; and $\mathrm{AP},+3.4$ $\mathrm{mm}, \mathrm{ML}, \pm 1.7 \mathrm{~mm}$, and DV,$-3.0 \mathrm{~mm}$, respectively. In each case the DV coordinate was relative to the surface of the thalamus at the injection site.

When the lesions were complete, the dura was repositioned but not sewn, the bone flap was replaced and held with loose sutures, and the skin and galea were closed in layers. The monkey was removed from the head-holder and anesthesia discontinued. Nonsteroidal antiinflammatory analgesic and antibiotic treatment continued after surgery in consultation with veterinary staff, typically for $5 \mathrm{~d}$.

Histology. After completion of all behavioral testing each monkey was sedated with ketamine $(10 \mathrm{mg} / \mathrm{kg})$, deeply anesthetized with intravenous barbiturate and transcardially perfused with $0.9 \%$ saline followed by $10 \%$ formalin. The brains were cryoprotected in formalin-sucrose and then sectioned coronally on a freezing microtome at $50 \mu \mathrm{m}$ thickness. A onein-five series of sections was collected throughout the thalamus; these were mounted on gelatin-coated glass microscope slides and stained with cresyl violet.

MDmc lesions. The three monkeys that formed the MDmc lesion group had extensive bilateral lesions in the magnocellular division of the mediodorsal thalamic nucleus as intended (Figs. 1,2). These lesions also damaged midline thalamic nuclei, namely the paraventricular and central intermedialis nuclei lying between the MDmc in the two hemispheres 
of the thalamus. This unavoidable damage alone could not have caused the new learning deficits (Gaffan and Murray, 1990), but in combination with the damage to MDmc may have contributed to the severity of the cognitive deficits during acquisition (see also Gaffan and Parker, 2000; Mitchell et al., 2007a). MD5 also sustained unilateral damage to the anterior thalamic nuclei that extended into the anterior portion of the laterodorsal thalamus. Other thalamic damage outside the MDmc was similar in all three lesions with slight damage to the borders of the rhomboid and central medial nuclei. The paraventricular nucleus of the epithalamus was extensively damaged throughout the entire anteroposterior extent of the lesions. All three monkeys also had sagittal section of the splenium of the corpus callosum and hippocampal commissure dorsal to the posterior thalamus.

\section{Results}

Monkeys destined to have bilateral neurotoxic lesions to MDmc or to remain as unoperated controls (CONs) did not differ in their ability to remember the scenes as measured by the total number of errors made in the preoperative one-trial retrieval test (Table 1). Monkeys with MDmc lesions, like the unoperated control monkeys, showed the same level of retention in the postoperative retrieval test as in the preoperative retrieval test. Thus, damage to the MDmc did not produce retrograde amnesia (Fig. 3, left). A 2 (group, control vs MDmc) $\times 2$ (testing phase, preoperative vs postoperative) $\times 3$ (set, three different sets of 100 scenes) repeated-measures ANOVA confirmed that there was no difference for lesion group or testing phase and no interaction of lesion group with any other factor (all $F$ values $<$ $1.0)$. There was only a significant difference for the retention levels of the three different sets $\left(F_{(2,12)}=\right.$ $44.13, p<0.001)$ that did not vary with lesion, reflecting the differing rates of training for the three sets of scenes during preoperative acquisition. Time spent completing the preoperative and postoperative one-trial retrieval tests did not differ for group, testing phase or set $(F$ values $<1.0)$.

Despite preserved memory of preoperatively learned scenes in the one-trial retrieval test, the monkeys with bilateral neurotoxic lesions to the MDmc had severe impairments in new learning. The MDmc lesion group were impaired in acquisition of 100 novel unique scene discriminations (set D) presented for learning in the same way as the preoperative sets, with one trial per scene per day. Monkeys with MDmc lesions accumulated more errors (Fig. 3, right) to reach the retention criterion of $85 \%$ correct across three consecutive trials (mean, 396.33; SD, 160.45) than unoperated controls (mean, 198.75; SD, 82.94), $\left(t_{(5)}=2.15, p=0.042\right.$, one-tailed). The MDmc group also required more trials to reach criterion (mean, 13.67; SD, 4.73) compared with controls (mean, 7.00; SD, 2.71), $\left(t_{(5)}\right.$ $=2.39, p<0.031$ ). Individual performance data for acquisition of set $\mathrm{D}$ are presented in Table 1.

\section{Discussion}

The present results demonstrate the dissociable contribution of the MDmc to memory acquisition
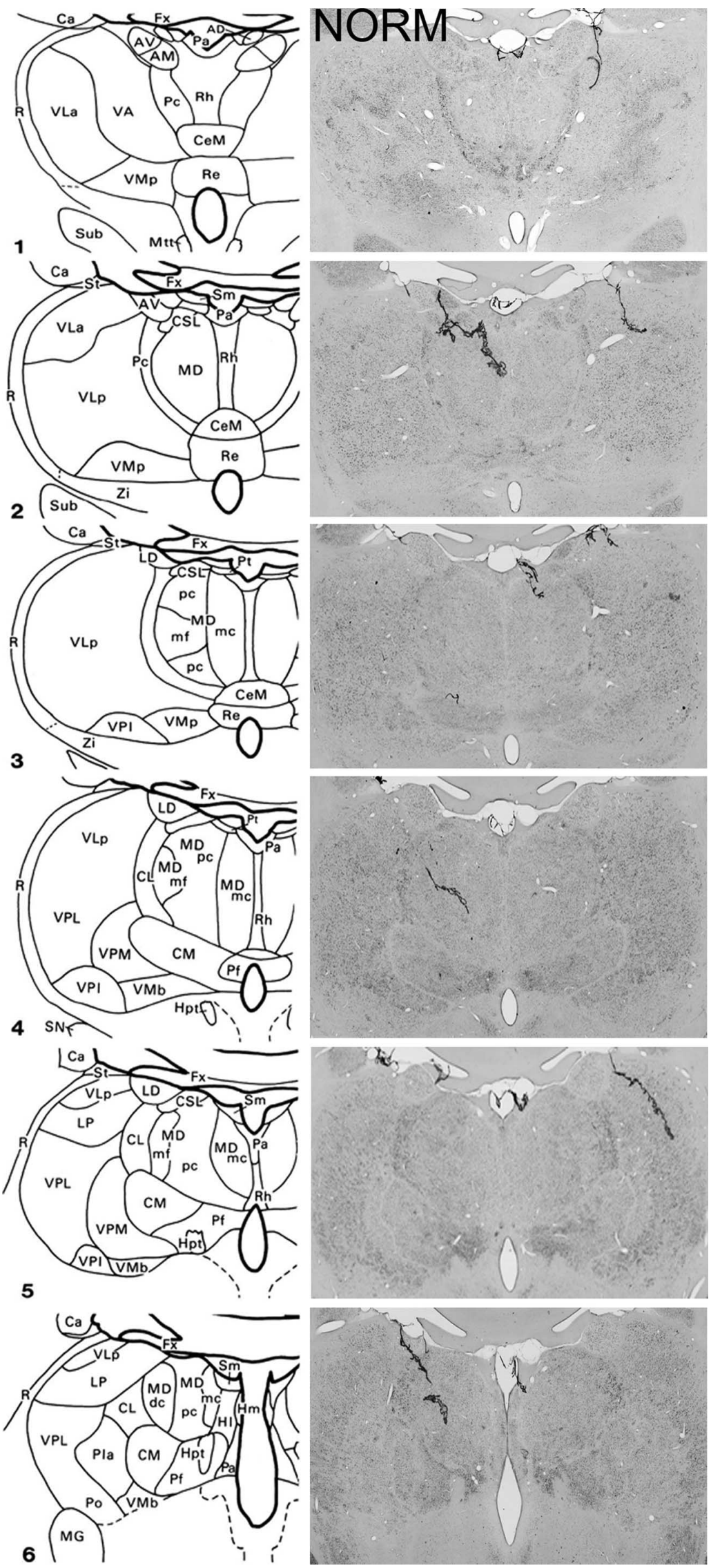

Figure 1. Schematic diagrams of six sections, $1 \mathrm{~mm}$ apart, through the medial thalamus of a monkey taken from Gaffan and Murray (1990) and photomicrographs of a normal medial thalamus (NORM) corresponding as closely as possible to the schematic diagrams. For abbreviations, see Mitchell et al. (2007a). 


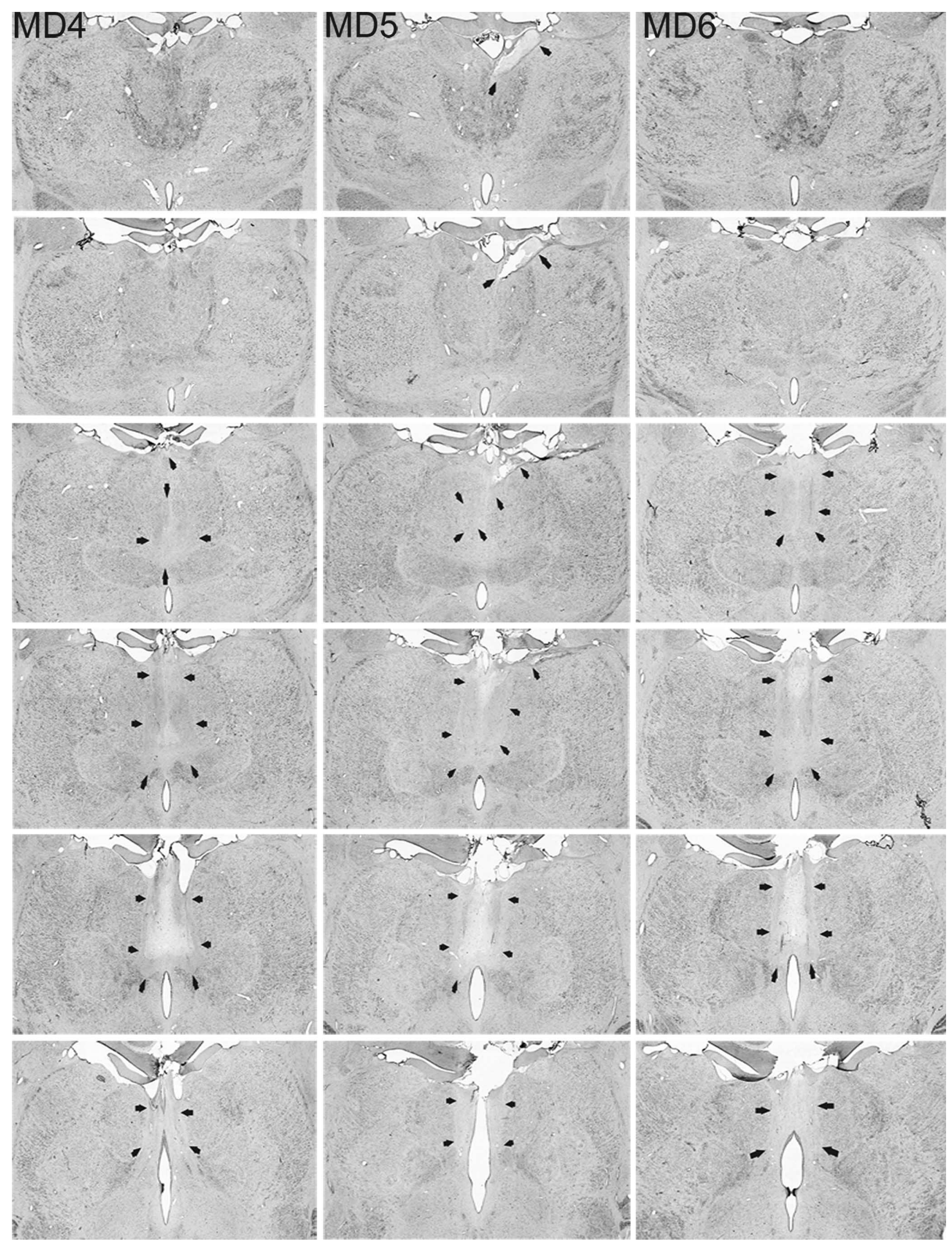

Figure 2. MDmc lesions. Photomicrographs of the MDmc lesions for MD4, MD5, and MD6 corresponding as closely as possible to normal medial thalamus (NORM) and the schematic diagrams of Figure 1 are shown. Arrows indicate the borders of each lesion. 
Table 1. Preoperative and Postoperative Retention and New Learning

\begin{tabular}{|c|c|c|c|c|c|c|c|c|c|}
\hline \multirow[b]{2}{*}{ Case } & \multirow{2}{*}{$\begin{array}{l}\text { Preoperative } \\
\text { Total Cycles of training to criterion }\end{array}$} & \multicolumn{3}{|c|}{ Preoperative $\%$ errors } & \multicolumn{3}{|c|}{ Postoperative $\%$ errors } & \multirow{2}{*}{\multicolumn{2}{|c|}{$\begin{array}{l}\text { Postoperative New Learning } \\
\text { Set D }\end{array}$}} \\
\hline & & Set A & Set B & Set $C$ & Set A & Set B & Set C & & \\
\hline & & & & & & & & Errors to criterion & Trials to criterion \\
\hline Con1 & 6 & 7 & 8 & 22 & 10 & 8 & 19 & 153 & 5 \\
\hline Con2 & 7 & 11 & 13 & 21 & 8 & 8 & 16 & 156 & 6 \\
\hline Con3 & 10 & 11 & 24 & 27 & 13 & 25 & 30 & 323 & 11 \\
\hline Con4 & 15 & 11 & 14 & 32 & 17 & 15 & 38 & - & - \\
\hline Con5 & 15 & 16 & 21 & 25 & 17 & 15 & 24 & 163 & 6 \\
\hline Mean & 10.6 & 11.2 & 16 & 25.4 & 13 & 14.2 & 25.4 & 198.8 & 7 \\
\hline MD4 & 8 & 17 & 15 & 28 & 14 & 12 & 30 & 337 & 12 \\
\hline MD5 & 15 & 19 & 15 & 26 & 18 & 19 & 24 & 274 & 10 \\
\hline MD6 & 10 & 10 & 18 & 27 & 15 & 26 & 28 & 578 & 19 \\
\hline Mean & 11 & 15.3 & 16 & 27 & 15.6 & 19 & 27.3 & 396.3 & 13.7 \\
\hline
\end{tabular}

The data shown are the total number of cycles of $6 \mathrm{~d}$ of training to reach the performance criterion of more than $85 \%$ correct across three consecutive trials of set $A$, the number of preoperative and postoperative retention errors made during the one-trial retrieval tests for set A (presented three times in every cycle of $6 \mathrm{~d}$ of training), set $\mathrm{B}$ (presented twice), and set C (presented once), and during postoperative new learning of set $\mathrm{D}$, the total number of errors made (excluding the first trial) and number of trials required to reach the criterion of 85\% across three consecutive trials for control monkeys (CON1-CON5) and monkeys with MDmc lesions (MD4-MD6).

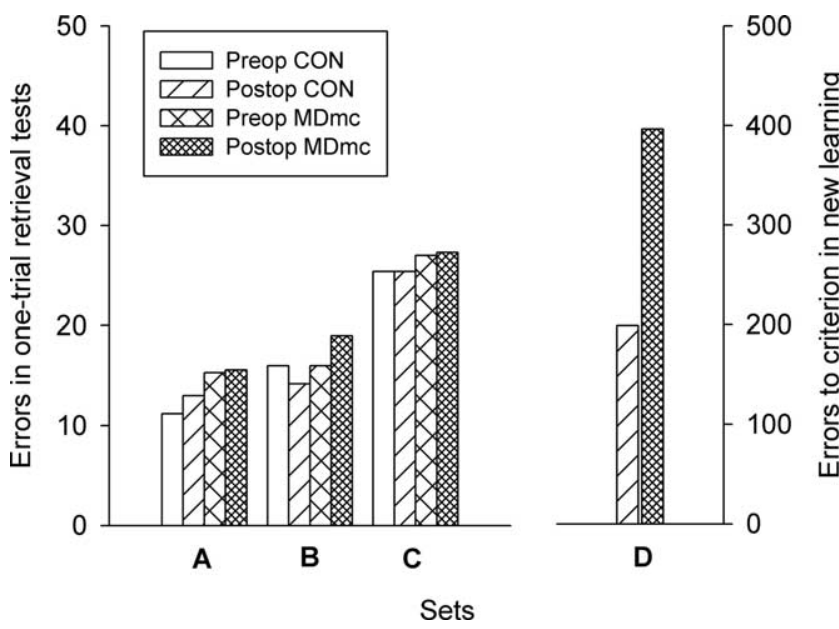

Figure 3. Preoperative and postoperative retention (left). The mean number of total errors made during the preoperative and postoperative one-trial retrieval tests of set $\mathrm{A}$ (presented three times in every cycle of $6 \mathrm{~d}$ of training during initial acquisition), set $B$ (presented twice), and set $C$ (presented once). New learning is shown on the right. The total number of errors made to reach the criterion of $85 \%$ correct across three consecutive trials during postoperative acquisition of 100 novel unique scene discriminations (set D) for neurotoxic magnocellular MDmc and unoperated control (CON) monkeys is shown.

and retrieval. Because the MDmc lesions only impaired new learning and not retention and retrieval, these data confirm the hypothesis that memory processes associated with retention and retrieval versus new learning involve differing interactions within the brain. Importantly, these results were produced using the same type of stimuli and testing conditions across the tests of retrieval and of new learning, and preoperatively monkeys destined to have MDmc lesions and unoperated controls did not differ in their acquisition rates of sets $\mathrm{A}$, $\mathrm{B}$, or C (see Table 1). Therefore, the impairment in new learning is not caused by other unrelated factors such as a perceptual, or other visual impairment, a lack of motivation, or an attentional deficit.

As the current data indicate, the MDmc is not critical for memory retrieval. Thus, it would appear that retrograde amnesia suffered in clinical cases of diencephalic amnesia is not the result of damage to the MDmc (Kopelman et al., 2003). In clinical cases, damage to the diencephalon is not simply confined to just one critical area but rather extends over many structures and nuclei that are important for memory processing (Victor et al., 1989; Kopelman, 1995; Harding et al., 2000; Van der Werf et al., 2003).
Bright et al. (2006) propose that frontal and temporal regions including lateral as well as medial temporal lobes form a neural network subserving retrieval whereby damage to the cortex needs to be quite extensive and exceed a certain critical volume before significant remote memory impairments can be observed. The current data also lend themselves to a similar conclusion whereby extensive neural damage in the medial diencephalon needs to occur before retrograde deficits are observed. For example, the mammillary bodies, the anterior thalamic nuclei, and lateral parts of the mediodorsal thalamic nucleus (i.e., the parvocellular and densocellular divisions) are also interconnected with the prefrontal cortex and temporal regions and the full extent of their involvement in retrograde and anterograde memory processes remains to be determined (Aggleton and Brown, 1999; Van der Werf et al., 2003).

Although the MDmc is not critical for memory retrieval, the current data support the proposal that damage to the MDmc contributes to the anterograde amnesia suffered in clinical cases of diencephalic amnesia. We theorize that interactions between MDmc and prefrontal cortex, specifically, are important for new learning of information, but not for retrieval of previously acquired information. The MDmc has prominent interconnections with the ventral and medial prefrontal cortex (Walker's areas 11, 12, 13, and 14) (Giguere and Goldman-Rakic, 1988; Ray and Price, 1993), lesions of which also impair new scene learning (Baxter et al., 2007; Wilson et al., 2007). Other recent data from our laboratory indicate that the fornix, which carries projections between medial temporal cortex and several subcortical structures, is also, like MDmc, more important for memory acquisition than for memory retrieval (Buckley et al., 2007). Many of the neurons that project through the fornix use neuromodulatory transmitters such as acetylcholine, which is believed on other grounds to be specifically important for memory acquisition (Tang et al., 1997). However, the reciprocal projections between MDmc and the prefrontal cortex are exclusively glutamatergic (Pirot et al., 1994). Thus, the present data suggest that corticalsubcortical glutamatergic interactions coursing between the prefrontal cortex and MDmc are, among other interactions including neuromodulatory interactions, more important for memory acquisition than for retrieval. Therefore in relation to the MDmc role in episodic-like memory tasks such as the object-in-place scene discrimination task, we propose that MDmc lesions disrupt the interactions between prefrontal cortex and the temporal lobes, which are all operating in concert to process memories. 


\section{References}

Aggleton JP, Mishkin M (1983) Memory impairments following restricted medial thalamic lesions in monkeys. Exp Brain Res 52:199-209.

Aggleton JP, Brown MW (1999) Episodic memory, amnesia, and the hippocampal-anterior thalamic axis. Behav Brain Sci 22:425-444.

Baxter MG, Gaffan D, Kyriazis DA, Mitchell AS (2007) Orbital prefrontal cortex is required for object-in-place scene memory but not performance of a strategy implementation task. J Neurosci 27:11327-11333.

Bright P, Buckman J, Fradera A, Yoshimasu H, Colchester AC, Kopelman MD (2006) Retrograde amnesia in patients with hippocampal, medial temporal, temporal lobe, or frontal pathology. Learn Mem 13:545-557.

Buckley MJ, Wilson CR, Gaffan D (2007) Fornix transection impairs visuospatial memory acquisition more than retrieval. Behav Neurosci, in press.

Gaffan D (1994) Scene-specific memory for objects: a model of episodic memory impairment in monkeys with fornix transection. J Cog Neurosci 6:305-320.

Gaffan D, Murray EA (1990) Amygdalar interaction with the mediodorsal nucleus of the thalamus and the ventromedial prefrontal cortex in stimulus reward associative learning in the monkey. J Neurosci 10:3479-3493.

Gaffan D, Parker A (2000) Mediodorsal thalamic function in scene memory in rhesus monkeys. Brain 123:816-827.

Gaffan D, Watkins S (1991) Mediodorsal thalamic lesions impair long-term visual associative memory in macaques. Eur J Neurosci 3:615-620.

Gaffan D, Murray EA, Fabrethorpe M (1993) Interaction of the Amygdala with the Frontal-Lobe in Reward Memory. Eur J Neurosci 5:968-975.

Giguere M, Goldman-Rakic PS (1988) Mediodorsal nucleus: areal, laminar, and tangential distribution of afferents and efferents in the frontal lobe of rhesus monkeys. J Comp Neurol 277:195-213.

Graff-Radford NR, Tranel D, Van Hoesen GW, Brandt JP (1990) Diencephalic amnesia. Brain 113:1-25.

Harding A, Halliday G, Caine D, Kril J (2000) Degeneration of anterior thalamic nuclei differentiates alcoholics with amnesia. Brain 123:141-154.

Ilinsky IA, Kultas-Ilinsky K (1987) Sagittal cytoarchitectonic maps of the Macaca mulatta thalamus with a revised nomenclature of the motorrelated nuclei validated by observations on their connectivity. J Comp Neurol 262:331-364.

Kopelman MD (1995) The Korsakoff syndrome. Br J Psychiatry 166:154-173.

Kopelman MD, Stanhope N, Kingsley D (1999) Retrograde amnesia in patients with diencephalic, temporal lobe or frontal lesions. Neuropsychologia 37:939-958.

Kopelman MD, Lasserson D, Kingsley DR, Bello F, Rush C, Stanhope N, Stevens TG, Goodman G, Buckman JR, Heilpern G, Kendall BE, Colchester AC (2003) Retrograde amnesia and the volume of critical brain structures. Hippocampus 13:879-891.
Mair WG, Warrington EK, Weiskrantz L (1979) Memory disorder in Korsakoff s psychosis: a neuropathological and neuropsychological investigation of two cases. Brain 102:749-783.

Mayes AR, Meudell PR, Mann D, Pickering A (1988) Location of lesions in Korsakoff's syndrome: neuropsychological and neuropathological data on two patients. Cortex 24:367-388.

Miller LA, Caine D, Harding A, Thompson EJ, Large M, Watson JD (2001) Right medial thalamic lesion causes isolated retrograde amnesia. Neuropsychologia 39:1037-1046.

Miller LA, Caine D, Watson JD (2003) A role for the thalamus in memory for unique entities. Neurocase 9:504-514.

Mitchell AS, Baxter MG, Gaffan D (2007a) Dissociable performance on scene memory learning and strategy implementation after lesions to magnocellular mediodorsal thalamic nucleus. J Neurosci 42:11888-11895.

Mitchell AS, Browning PG, Baxter MG (2007b) Neurotoxic lesions of the medial mediodorsal nucleus of the thalamus disrupt reinforcer devaluation effects in rhesus monkeys. J Neurosci 42:11289-11295.

Parker A, Gaffan D (1998) Interaction of frontal and perirhinal cortices in visual object recognition memory in monkeys. Eur J Neurosci 10:3044-3057.

Parker A, Eacott MJ, Gaffan D (1997) The recognition memory deficit caused by mediodorsal thalamic lesion in non-human primates: a comparison with rhinal cortex lesion. Eur J Neurosci 9:2423-2431.

Parkin AJ, Rees JE, Hunkin NM, Rose PE (1994) Impairment of memory following discrete thalamic infarction. Neuropsychologia 32:39-51.

Pirot S, Jay TM, Glowinski J, Thierry AM (1994) Anatomical and electrophysiological evidence for an excitatory amino acid pathway from the thalamic mediodorsal nucleus to the prefrontal cortex in the rat. Eur J Neurosci 6:1225-1234.

Ray JP, Price JL (1993) The organization of projections from the mediodorsal nucleus of the thalamus to orbital and medial prefrontal cortex in macaque monkeys. J Comp Neurol 337:1-31.

Tang Y, Mishkin M, Aigner TG (1997) Effects of muscarinic blockade in perirhinal cortex during visual recognition. Proc Natl Acad Sci USA 94:12667-12669.

Van der Werf YD, Jolles J, Witter MP, Uylings HB (2003) Contributions of thalamic nuclei to declarative memory functioning. Cortex 39:1047-1062.

Victor M, Adams RD, Collins GH (1989) The Wernicke-Korsakoff syndrome, Ed 2. Philadelphia: F. A. Davis.

Wilson CR, Gaffan D, Mitchell AS, Baxter MG (2007) Neurotoxic lesions of ventrolateral prefrontal cortex impair object-in-place scene memory. Eur J Neurosci 25:2514-2522.

Zola-Morgan S, Squire LR (1985) Amnesia in monkeys after lesions of the mediodorsal nucleus of the thalamus. Ann Neurol 17:558-564. 